\title{
An Efficient Algorithm for Finding the Maximal Eigenvalue of Zero Symmetric Nonnegative Matrices
}

\author{
Gang Wang (D) and Lihong Sun \\ School of Management Science, Qufu Normal University, Rizhao, Shandong, 276800, China \\ Correspondence should be addressed to Gang Wang; wgglj1977@163.com
}

Received 27 July 2018; Revised 6 November 2018; Accepted 12 November 2018; Published 29 November 2018

Academic Editor: Alessandro Formisano

Copyright (c) 2018 Gang Wang and Lihong Sun. This is an open access article distributed under the Creative Commons Attribution License, which permits unrestricted use, distribution, and reproduction in any medium, provided the original work is properly cited.

In this paper, we propose an improved power algorithm for finding maximal eigenvalues. Without any partition, we can get the maximal eigenvalue and show that the modified power algorithm is convergent for zero symmetric reducible nonnegative matrices. Numerical results are reported to demonstrate the effectiveness of the modified power algorithm. Finally, a modified algorithm is proposed to test the positive definiteness (positive semidefiniteness) of $Z$-matrices.

\section{Introduction}

The maximum eigenvalue problems of nonnegative matrices are important tools for matrix analysis and calculation and are widely used in many fields, such as principal component analysis, Markov chain [1], and stability of control systems [2-7]. Some researchers directly estimated the bounds of the maximum eigenvalues according to the nature of the nonnegative irreducible matrices [8-11]. Based on a geometric symmetrization of powers of matrix, Szyld [10] presented a sequence of lower bounds for the spectral radius. Dursun et al. [8] gave a sequence of upper bounds for maximal eigenvalue of a nonnegative matrix, which complemented results of [10]. Due to the complexity of the maximal eigenvalue problem, it is difficult to obtain exact solutions by means of estimation methods. Hence, some efficient algorithms for computing eigenpairs of nonnegative matrices have been proposed such as (improved) Arnoldi's algorithm $[12,13]$, (modified) diagonal transformation algorithm [1417], and power algorithm [18-24]. Generally, (improved) Arnoldi's method [12] is suitable for computing some selected eigenpairs of large asymmetrical matrices. The (modified) diagonal transformation algorithm is convergent for irreducible nonnegative matrices $[15,17]$. Wood et al. [22, 23] proposed the convergent power method to calculate maximum eigenvalue of irreducible nonnegative matrices. For reducible matrices, the diagonal transformation algorithm and power algorithm may not be convergent $[15,22]$. As we know, reducible matrices can be decomposed into some irreducible matrices. However, it is very costly to test the irreducibility and determine the partition for large-size matrices. Motivated by these observations, we want to establish a modified power algorithm and obtain the maximal eigenvalue of zero symmetric reducible nonnegative matrices without any partition.

This paper is organized as follows. In Section 2, we introduce important notation and recall fundamental results. In Section 3, we propose a modified power algorithm for computing the maximal eigenvalue of zero symmetric reducible nonnegative matrices. It is worth noting that the algorithm is convergent and does not require the partition for zero symmetric reducible nonnegative matrices. Numerical results are reported to demonstrate the effectiveness of the proposed algorithm. As an application, we can test the positive definiteness (positive semidefiniteness) of $Z$-matrices in Section 4.

\section{Notation and Preliminaries}

We start this section with some fundamental notion and properties of a nonnegative matrix, which are needed in the subsequent analysis. 
Definition 1. (i) A matrix $A=\left(a_{i j}\right)_{n \times n} \in R^{n, n}$ is called symmetric if

$$
a_{i j}=a_{j i}
$$
if

(ii) A matrix $A=\left(a_{i j}\right)_{n \times n} \in R^{n, n}$ is called zero symmetric

$$
\begin{aligned}
a_{i j} & =0 \Longrightarrow \\
a_{j i} & =0 \\
\text { and } a_{j i} & =0 \Longrightarrow \\
a_{i j} & =0
\end{aligned}
$$

Obviously, if a matrix $A$ is symmetric, then $A$ is zero symmetric. Conversely, the result may not hold.

Definition 2. A matrix $A \in R^{n, n}$ is said to be reducible if there exists a permutation matrix $P \in R^{n, n}$ such that

$$
P A P^{\mathrm{T}}=\left(\begin{array}{cc}
A_{11} & 0 \\
A_{21} & A_{22}
\end{array}\right),
$$

where $A_{11} \in R^{r, r}, A_{22} \in R^{n-r, n-r}, A_{21} \in R^{n-r, r}, 1 \leq r \leq$ $n-1$, and $0 \in R^{r, n-r}$ is the zero matrix. If such a permutation matrix does not exist, $A$ is called irreducible.

Definition 3. Let $A=\left(a_{i j}\right)$ be a nonnegative matrix of dimension $n$ and $I \subset\{1, \ldots, n\}$ with $|I|=r$. A submatrix $A_{I}$ of the matrix $A$ with index set $I$ is an $r$-dimensional submatrix of $A$ consisting of $r^{2}$ elements defined as follows:

$$
A_{I}=\left(d_{i j}\right)=a_{i j}, \quad i, j \in I,
$$

where $|I|$ is the number of elements of $I$.

Definition 4. We call $\sigma(A)$ the set of all eigenvalues of $A$. Assume $\sigma(\mathscr{A}) \neq \emptyset$. Then the spectral radius of $A$ is denoted by

$$
\rho(A)=\max \{|\lambda|: \lambda \in \sigma(A)\} .
$$

If $A$ is a nonnegative matrix, then $\rho(A)$ is the maximal eigenvalue.

Let $P_{n}=\left\{x \in R^{n}: x_{i} \geq 0,1 \leq i \leq n\right\}$ and $\operatorname{int}\left(P_{n}\right)=\{x \in$ $\left.R^{n}: x_{i}>0,1 \leq i \leq n\right\}$. For any nonnegative column vector $x \in R^{n}$, we define $\phi: P_{n} \longrightarrow P_{1}$ by

$$
\phi(x)=\sum_{i=1}^{n} x_{i} .
$$

Now, we give the power algorithm [22, 23] of irreducible nonnegative matrices as follows.

Algorithm 5.

Step 1. Choose $x^{(1)} \in \operatorname{int}\left(P_{n}\right), \alpha>0, \epsilon>0$, and a unit matrix $I, k=1$.
Step 2. Compute

$$
\begin{aligned}
y^{(k)} & =(A+\alpha I) x^{(k)}, \\
\bar{\lambda}_{k} & =\max _{1 \leq i \leq n} \frac{\left(y^{(k)}\right)_{i}}{\left(x^{(k)}\right)_{i}} \\
\underline{\lambda}_{k} & =\min _{1 \leq i \leq n} \frac{\left(y^{(k)}\right)_{i}}{\left(x^{(k)}\right)_{i}} .
\end{aligned}
$$

Step 3. If $\bar{\lambda}_{k}-\underline{\lambda}_{k}<\epsilon$, stop. Output the maximal eigenvalue $\rho(A)=(1 / 2)\left(\bar{\lambda}_{k}+\underline{\lambda}_{k}\right)-\alpha$. Otherwise, set $k=k+1$ and compute

$$
x^{(k+1)}=\frac{y^{(k)}}{\phi\left(y^{(k)}\right)},
$$

and go to Step 2 .

For irreducible nonnegative matrices, we have the following convergent theorems.

Lemma 6 (Theorem 9 of [22]). If $A$ is an irreducible nonnegative matrix, then the matrix $q I+A$ is primitive, where $q>0$ and $I$ is a unit matrix.

Lemma 7 (Theorem 8 of [22]). $\left\{\bar{\lambda}_{k}\right\},\left\{\underline{\lambda}_{k}\right\}$ generated by Algorithm 5 converge to $\rho(A)$, if and only if the irreducible nonnegative matrix $A$ is primitive.

\section{Modified Power Algorithm for the Maximal Eigenvalue of Zero Symmetric Nonnegative Matrices}

In this section, we give the modified power algorithm for computing the maximal eigenvalue of zero symmetric reducible nonnegative matrices without any partition. Furthermore, we demonstrate that this algorithm is convergent by Theorem 10. We state the algorithm as follows.

Algorithm 8.

Step 1. Choose $x^{(1)} \in \operatorname{int}\left(P_{n}\right)$, and $\alpha>0, \epsilon>0$, and $k=1$.

Step 2. Compute

$$
\begin{aligned}
y^{(k)} & =(A+\alpha I) x^{(k)}, \\
\bar{\lambda}_{k} & =\max _{1 \leq i \leq n} \frac{\left(y^{(k)}\right)_{i}}{\left(x^{(k)}\right)_{i}} .
\end{aligned}
$$

Step 3. If $\left(\bar{\lambda}_{k-1}-\bar{\lambda}_{k}\right)<\epsilon$, stop. Output the maximal eigenvalue $\rho(A)=\bar{\lambda}_{k}-\alpha$. Otherwise, set $k=k+1$ and compute

$$
x^{(k+1)}=\frac{y^{(k)}}{\phi\left(y^{(k)}\right)},
$$

and go to Step 2 . 
We show that the sequence $\left\{\bar{\lambda}_{k}\right\}$ generated by Algorithm 8 is convergent and the limit is the maximal eigenvalue for any zero symmetric nonnegative matrices. In what follows, we give the following lemma.

Lemma 9. Let $A=\left(a_{i j}\right)_{n \times n}$ be a zero symmetric reducible nonnegative matrix. Then, we have the following results:

(I) There exists a partition $\left\{I_{1}, I_{2}, \ldots, I_{s}\right\}$ of $\{1,2, \ldots, n\}$ such that each induced matrix $A_{I_{i}}, i=1,2, \ldots, s$, either is irreducible or is a zero matrix

(II) For $x \in R^{n}$, there exists an $n \times n$ permutation $P$ such that

$$
P(A x)=\left(\begin{array}{c}
A_{I_{1}} x_{I_{1}} \\
A_{I_{2}} x_{I_{2}} \\
\vdots \\
A_{I_{k}} x_{I_{s}}
\end{array}\right)
$$

(III) $\rho(A)=\max _{1 \leq i \leq s} \rho\left(A_{I_{i}}\right)$.

For a zero symmetric reducible nonnegative matrix $A$, by Lemma 9, it holds that

$$
\rho(A)=\max \left\{\rho\left(A_{I_{1}}\right), \rho\left(A_{I_{i}}\right), \ldots, \rho\left(A_{I_{s}}\right)\right\}
$$

As we know, it is very costly to determine the partition for large-size reducible matrices. Fortunately, Algorithm 8 is not necessary to decompose the matrix and can compute the maximal eigenvalue of each block matrix.

Theorem 10. Suppose that $A$ is a zero symmetric reducible nonnegative matrix and the sequence $\left\{\bar{\lambda}_{k}\right\}$ is generated by Algorithm 8. Then, $\rho(A)=\lim _{k \rightarrow \infty} \bar{\lambda}_{k}$.

Proof. For the sequence $\left\{\bar{\lambda}_{k}\right\}$, it holds that $\left\{\bar{\lambda}_{k}\right\}$ is monotonously decreasing and bounded to the below. According to Algorithm 8 , it is easy to get $\bar{\lambda}_{k} \geq \alpha$. Now, we show that $\left\{\bar{\lambda}_{k}\right\}$ is a decreasing sequence. For any $k \geq 1$, by Algorithm 8 , we have

$$
y^{(k)}=(A+\alpha I) x^{(k)} \leq \bar{\lambda}_{k} x^{(k)}
$$

So,

$$
x^{(k+1)}=\frac{y^{(k)}}{\phi\left(y^{(k)}\right)} \leq \frac{\bar{\lambda}_{k} x^{(k)}}{\phi\left(y^{(k)}\right)} .
$$

Since $A$ is nonnegative, we get

$$
\begin{aligned}
(A+\alpha I) x^{(k+1)} & \leq \frac{(A+\alpha I)\left(\bar{\lambda}_{k} x^{(k)}\right)}{\phi\left(y^{(k)}\right)} \\
& =\frac{\bar{\lambda}_{k}(A+\alpha I)\left(x^{(k)}\right)}{\phi\left(y^{(k)}\right)}=\frac{\bar{\lambda}_{k} y^{(k)}}{\phi\left(y^{(k)}\right)} \\
& =\bar{\lambda}_{k} x^{(k+1)} .
\end{aligned}
$$

For $i=1,2, \cdots, n$, one has

$$
\bar{\lambda}_{k} \geq \max _{1 \leq i \leq n} \frac{\left((A+\alpha I) x^{(k+1)}\right)_{i}}{\left(x^{(k+1)}\right)_{i}}=\max _{1 \leq i \leq n} \frac{\left(y^{(k+1)}\right)_{i}}{\left(x^{(k+1)}\right)_{i}}=\bar{\lambda}_{k+1},
$$

which means that $\left\{\bar{\lambda}_{k}\right\}$ is a decreasing sequence. So, the sequence $\left\{\bar{\lambda}_{k}\right\}$ is convergent. Hence, there exists $\bar{\lambda}$ such that $\bar{\lambda}_{k} \longrightarrow \bar{\lambda}$ when $k \longrightarrow \infty$. Next, we show that $\bar{\lambda}=\rho(\mathrm{A})+\alpha$. Without loss of generality, we assume that the matrix $A+\alpha I$ is stated as follows:

$$
A+\alpha I
$$

$$
=\left(\begin{array}{cccc}
A_{I_{1}}+\alpha I_{I_{1}} & 0 & \cdots & 0 \\
0 & A_{I_{2}}+\alpha I_{I_{2}} & \cdots & 0 \\
\vdots & \vdots & \ddots & \vdots \\
0 & 0 & \cdots & A_{I_{s}}+\alpha I_{I_{s}}
\end{array}\right) \text {, }
$$

where each block $A_{I_{i}}+\alpha I_{I_{i}}(i=1,2, \ldots, s)$ is square and either is primitive or is a $1 \times 1$ unit matrix. It follows from Lemma 9 that

$$
\rho(A)=\max \left\{\rho\left(A_{I_{1}}\right), \rho\left(A_{I_{2}}\right), \ldots, \rho\left(A_{I_{s}}\right)\right\} .
$$

We divide the proof into two parts.

Case 1. A has a unique maximal eigenvalue. Without loss of generality, we assume

$$
\begin{array}{r}
\rho(A)=\rho\left(A_{I_{1}}\right)>\max \left\{\rho\left(A_{I_{2}}\right), \ldots, \rho\left(A_{I_{s}}\right)\right\} \\
(i=2,3, \ldots, s) .
\end{array}
$$

Let $\bar{\lambda}_{k}$ be the maximum value of matrix $A+\alpha I$ generated by Algorithm 8 . Let $\left(\bar{\lambda}_{k}^{i},\left(x^{(k)}\right)_{I_{i}}\right)(i=1,2, \ldots, s)$ be the maximum value and eigenvector of $\left(A_{I_{i}}+\alpha I_{I_{i}}\right)$ and let $\rho\left(A_{I_{i}}\right)$ be the maximum eigenvalue of $A_{I_{i}}(i=1,2, \ldots, s)$. Set

$$
\beta_{i}=\rho\left(A_{I_{1}}\right)-\rho\left(A_{I_{i}}\right) \quad(i=2,3, \ldots, s) .
$$

Obviously, $\beta_{i}>0$. Taking into account that each block $A_{I_{i}}+$ $\alpha I_{I_{i}}$ is primitive, from Lemma 7, we have

$$
\lim _{k \rightarrow \infty} \bar{\lambda}_{k}^{i}=\rho\left(A_{I_{i}}\right)+\alpha \quad(i=1,2, \ldots, s) .
$$

Since $A_{I_{1}}+\alpha I_{I_{1}}$ is primitive and $x^{(k+1)}=y^{(k)} / \phi\left(y^{(k)}\right)$, from Algorithm 8, we obtain

$$
\left.\left(y^{(k)}\right)_{I_{1}}=\left(A_{I_{1}}+\alpha I_{I_{1}}\right)\left(x^{(k)}\right)_{I_{1}}\right)>0 .
$$

For $0<\epsilon<\min _{2 \leq i \leq s} \beta_{i}, \exists K$, when $k>K$, we have

$$
\left|\bar{\lambda}_{k}^{i}-\rho\left(A_{I_{i}}\right)-\alpha\right|<\epsilon
$$

and, furthermore,

$$
\rho\left(A_{I_{i}}\right)+\alpha+\epsilon>\bar{\lambda}_{k}^{i}, \quad \forall i=1,2, \ldots, s .
$$


On the other hand, since $\rho\left(A_{I_{1}}\right)-\rho\left(A_{I_{i}}\right)=\beta_{i}$ and $0<\epsilon<\beta_{i}$, it holds that

$$
\rho\left(A_{I_{1}}\right)+\alpha>\rho\left(A_{I_{i}}\right)+\alpha+\epsilon, \quad \forall i=2, \ldots, s .
$$

For $k>K$, it follows from (24) that

$$
\bar{\lambda}_{k}^{1} \geq \rho\left(A_{I_{1}}\right)+\alpha>\bar{\lambda}_{k}^{i}, \quad \forall i=2, \ldots, s
$$

and, equivalently,

$$
\bar{\lambda}_{k}^{1}>\bar{\lambda}_{k}^{i}, \quad \forall i=2, \ldots, s .
$$

It follows from $\bar{\lambda}_{k}=\max \left\{\bar{\lambda}_{k}^{1}, \bar{\lambda}_{k}^{2}, \ldots, \bar{\lambda}_{k}^{s}\right\}$ that

$$
\bar{\lambda}_{k}^{1}=\bar{\lambda}_{k}, \quad \forall k>K
$$

From Lemma 7, we obtain

$$
\rho\left(A_{I_{1}}\right)+\alpha=\lim _{k \rightarrow \infty} \bar{\lambda}_{k}^{1}=\lim _{k \rightarrow \infty} \bar{\lambda}_{k}=\rho(A)+\alpha .
$$

Case 2. A has two maximal eigenvalues, that is,

$$
\begin{aligned}
& \rho(A)= \rho\left(A_{I_{1}}\right)=\rho\left(A_{I_{2}}\right) \\
&>\max \left\{\rho\left(A_{I_{1}}\right), \rho\left(A_{I_{2}}\right), \ldots, \rho\left(A_{I_{s}}\right)\right\} \\
& \\
& \quad(i=3,4, \ldots, s) .
\end{aligned}
$$

According to the above analysis, $\exists K$, when $k>K$, we know

$$
\begin{aligned}
& \bar{\lambda}_{k}^{1}>\bar{\lambda}_{k}^{i} \text { or } \\
& \bar{\lambda}_{k}^{2}>\bar{\lambda}_{k}^{i},
\end{aligned}
$$

$$
i=3,4, \ldots, s \text {. }
$$

For $k>K$, we deduce $\bar{\lambda}_{k}^{1}=\bar{\lambda}_{k}$ or $\bar{\lambda}_{k}^{2}=\bar{\lambda}_{k}$. It follows from Lemma 7 that

$$
\begin{aligned}
& \lim _{k \rightarrow \infty} \bar{\lambda}_{k}^{1}=\rho\left(A_{I_{1}}\right)+\alpha \text { or } \\
& \lim _{k \rightarrow \infty} \bar{\lambda}_{k}^{2}=\rho\left(A_{I_{2}}\right)+\alpha,
\end{aligned}
$$

and, equivalently,

$$
\begin{aligned}
& \rho\left(A_{I_{1}}\right)+\alpha=\lim _{k \rightarrow \infty} \bar{\lambda}_{k}^{1}=\lim _{k \rightarrow \infty} \bar{\lambda}_{k}=\rho(A)+\alpha \text { or } \\
& \rho\left(A_{I_{2}}\right)+\alpha=\lim _{k \rightarrow \infty} \bar{\lambda}_{k}^{2}=\lim _{k \rightarrow \infty} \bar{\lambda}_{k}=\rho(A)+\alpha .
\end{aligned}
$$

When $A$ has more than two maximal eigenvalues, we repeat the above process and can obtain the same convergent conclusions.
Remark 11. We are able to obtain an eigenvector corresponding to $\rho(A)$ as follows. Since the sequence $\left\{x^{(k)}\right\}$ is bounded, it has an accumulation point. Suppose that $x^{*}$ is an accumulation point of $\left\{x^{(k)}\right\}$. Define $\bar{x}^{*}$ by

$$
\bar{x}_{j}^{*}=\left\{\begin{array}{ll}
x_{j}^{*}, & j \in I^{*}, \\
0, & \text { otherwise, }
\end{array} \quad j=1, \ldots, n,\right.
$$

where $I^{*}=\left\{1 \leq j \leq n:\left(A x^{*}\right)_{j}=\rho(A) x_{j}^{*}\right.$ and $\left.x_{j}^{*}>0\right\}$. By Lemma $9, \bar{x}^{*}$ is an eigenvector corresponding to $\rho(\mathscr{A})$ when $\bar{x}^{*} \neq 0$.

In the following, we report numerical results for Algorithm 8. In order to show this algorithm is efficient, we compare it with improved Arnoldi's algorithm in [13] and the modified diagonal algorithm in [17]. For Algorithm 8, we stop the iteration as long as $\left|\bar{\lambda}_{k-1}-\bar{\lambda}_{k}\right| \leq \varepsilon, \varepsilon=10^{-10}$. (Detailed information is in Tables 1 and 2.)

All testing $n \times n$ matrices $B$ are generated as follows: Give an integer $p$ and generate randomly three positive matrices $B_{I_{1}}, B_{I_{2}}, B_{I_{3}}$. Let $I_{1}=\{1,2, \ldots, p\}, I_{2}=\{p+1, \ldots, 2 p\}$, and $I_{3}=$ $\{2 p+1, \ldots, 3 p\}$. Then, define $B=\left(B_{I_{1}}, B_{I_{2}}, B_{I_{3}}\right)$, where $n=$ $3 p$, other elements being zero. Clearly, $B$ is zero symmetric reducible. Algorithm 8 is implemented in MATLAB (R2011a) and all the numerical computations are conducted using an Intel $3.60 \mathrm{GHz}$ computer with $8 \mathrm{~GB}$ of RAM. In Table 1 , the CPU time is the average of 10 instances for each $n$. From Table 1, we can see that both algorithms can find maximal eigenvalues of the testing matrices but Algorithm 8 uses much less CPU time than improved Arnoldi's algorithm in [13], especially for large-size matrices.

Considering that the matrix is nonnegative and irreducible for the modified diagonal algorithm in [17], all testing matrices $B$ are positive matrices generated randomly in Table 2. Compared with the modified diagonal algorithm in [17], the Algorithm 8 has better execution efficiency in CPU time.

\section{Applications}

In this section, we give an application of Algorithm 8 for testing the positive definiteness (positive semidefiniteness) for large-size $Z$-matrices. We recall some definitions and notations about $Z$-matrices and $M$-matrices.

Definition 12. A matrix $A \in R^{n, n}$ is called a $Z$-matrix if it can be written as $A=c I-B$, where $I$ is a unit matrix and $\mathscr{B}$ is a nonnegative matrix. Furthermore, if $c \geq \rho(B)$, then $A$ is said to be an $M$-matrix; if $c>\rho(B)$, then $A$ is said to be a strong $M$-matrix.

Let $A \in R^{n, n}$ and $x \in R^{n}$. Define a quadratic polynomial form $f_{A}(x)$ as follows:

$$
f_{A}(x):=\sum_{i, j=1}^{n} a_{i j} x_{i} x_{j} .
$$

Definition 13. (i) $A$ is called positive definite if $f_{A}(x)>0$ for all $x \in R^{n}, x \neq 0$. 
TABLE 1: Comparisons of Algorithm 8 and improved Arnoldi's algorithm in [13].

\begin{tabular}{lccc}
\hline & & Algorithm 8 & Improved Arnoldi Algorithm \\
\hline $\mathrm{n}$ & $\rho$ & $c p u(s)$ & $c p u(s)$ \\
\hline 600 & $2.5024 \mathrm{e}+02$ & 0.032054 & 0.129545 \\
\hline 1200 & $9.9961 \mathrm{e}+02$ & 0.092272 & 0.225116 \\
\hline 1800 & $1.4999 \mathrm{e}+03$ & 0.186048 & 0.400719 \\
\hline 3000 & $2.0000 \mathrm{e}+03$ & 0.295846 & 0.678812 \\
\hline 6000 & $2.5001 \mathrm{e}+03$ & 0.459340 & 0.990805 \\
\hline 9000 & $3.9026 \mathrm{e}+03$ & 1.248525 & 2.255433 \\
\hline 12000 & $5.0004 \mathrm{e}+03$ & 3.812981 & 5.945294 \\
\hline
\end{tabular}

TABLE 2: Comparisons of Algorithm 8 and the modified diagonal algorithm in [17].

\begin{tabular}{lccc}
\hline & & Algorithm 8 & Modified diagonal algorithm \\
\hline $\mathrm{n}$ & $\rho$ & $c p u(s)$ & $c p u(s)$ \\
\hline 500 & $5.0033 \mathrm{e}+02$ & 0.030557 & 1.207591 \\
\hline 1000 & $1.0007 \mathrm{e}+03$ & 0.089176 & 7.937777 \\
\hline 1500 & $1.4994 \mathrm{e}+03$ & 0.154085 & 21.985712 \\
\hline 2000 & $2.0000 \mathrm{e}+03$ & 0.263202 & 47.984927 \\
\hline 3000 & $2.4994 \mathrm{e}+03$ & 0.415304 & 91.207171 \\
\hline
\end{tabular}

(ii) $A$ is called positive semidefinite if $f_{A}(x) \geq 0$ for all $x \in R^{n}$

As we know, for a symmetric matrix $A, A$ is positive definite if and only if all of its eigenvalues are positive, and $A$ is positive semidefinite if and only if all of its eigenvalues are nonnegative. For an asymmetric $Z$-matrix $A$, we can do the following transformation to verify whether $A$ is a positive definite (semidefinite) matrix.

For a $Z$-matrix $A \in R^{n, n}$, consider the following optimization problem:

$$
v^{*}=\min \left\{f_{A}(x): \sum_{i=1}^{n} x_{i}^{2}=1\right\}
$$

Clearly, we observe that $A$ is positive definite if and only if $v^{*}>0$, and $A$ is positive semidefinite if and only if $v^{*} \geq 0$. Set

$$
\begin{aligned}
& \bar{c}=\max \left\{a_{i i}: 1 \leq i \leq n\right\}, \\
& \underline{c}=\min \left\{a_{i i}: 1 \leq i \leq n\right\} .
\end{aligned}
$$

Then, $A$ can be written as $A=\bar{c} I-B$, where $B$ is a nonnegative matrix.

Given indices $r_{1}, r_{2}$, where $r_{1}, r_{2}=1,2, \ldots, n$, let $S_{\left(r_{1}, r_{2}\right)}$ be the set of all permutations of the indices $r_{1}, r_{2}$. For any $\pi\left(r_{1}, r_{2}\right) \in S_{\left(r_{1}, r_{2}\right)}$, let

$$
\bar{b}_{\pi\left(r_{1}, r_{2}\right)}=\frac{1}{2} \sum_{\sigma\left(r_{1}, r_{2}\right) \in S\left(r_{1}, r_{2}\right)} b_{\sigma\left(r_{1}, r_{2}\right)}
$$

and define

$$
\bar{B}=\left(\bar{b}_{i j}\right), \quad 1 \leq i, j \leq n
$$

Hence, $\bar{B}$ is a symmetrized matrix of $B$. For any $x \in R^{n}$, set

$$
\begin{aligned}
& f_{B}(x):=\sum_{i, j=1}^{n} b_{i j} x_{i} x_{j}, \text { and } \\
& f_{\bar{B}}(x):=\sum_{i, j=1}^{n} \bar{b}_{i j} x_{i} x_{j} .
\end{aligned}
$$

Clearly, we have that $f_{\bar{B}}(x)=f_{B}(x)$. Therefore,

$$
\begin{aligned}
v^{*} & =\min \left\{f_{A}(x): \sum_{i=1}^{n} x_{i}^{2}=1\right\} \\
& =\min \left\{f_{(\bar{c} I-B)}(x): \sum_{i=1}^{n} x_{i}^{2}=1\right\} \\
& =\min \left\{\bar{c}-f_{B}(x): \sum_{i=1}^{n} x_{i}^{2}=1\right\} \\
& =\min \left\{\bar{c}-f_{\bar{B}}(x): \sum_{i=1}^{n} x_{i}^{2}=1\right\} \\
& =\bar{c}-\max \left\{f_{\bar{B}}(x): \sum_{i=1}^{n} x_{i}^{2}=1\right\}=\bar{c}-\rho(\bar{B}) .
\end{aligned}
$$

From the above analysis, we can propose the following algorithm for testing the positive definiteness (positive semidefiniteness) of $Z$-matrices. 
Algorithm 14.

Step 1. Given a $Z$-matrix $A \in R^{n, n}$, compute

$$
\begin{aligned}
& \bar{c}=\max \left\{a_{i i}: 1 \leq i \leq n\right\}, \\
& \underline{c}=\min \left\{a_{i i}: 1 \leq i \leq n\right\} .
\end{aligned}
$$

If $\underline{c}<0$, then $A$ is not positive semidefinite and stop. Otherwise, go to the next step.

Step 2. Let $B=\bar{c} I-A$ and compute its symmetrized matrix $\bar{B}$. By Algorithm 8, compute the spectral radius $\rho(\bar{B})$.

Step 3. Let $v^{*}=\bar{c}-\rho(\bar{B})$. If $v^{*}>0, A$ is positive definite. If $v^{*} \geq 0, A$ is positive semidefinite. Otherwise, it is indefinite.

\section{Conclusions}

In this paper, a modified power algorithm was proposed for finding the maximal eigenvalue of zero symmetric nonnegative matrices. The modified power algorithm has the following important properties: (1) the convergence property is guaranteed for any zero symmetric nonnegative matrices and (2) it gets the maximal eigenvalue without any partition. Finally, we proposed Algorithm 14 to test the positive definiteness (positive semidefiniteness) of $Z$-matrices.

\section{Data Availability}

The data used to support the findings of this study are included within the article.

\section{Conflicts of Interest}

The authors declare that they have no conflicts of interest.

\section{Acknowledgments}

This research is supported by the Natural Science Foundation of China (11671228) and the Natural Science Foundation of Shandong Province (ZR2016AM10).

\section{References}

[1] R. A. Horn and C. R. Johnson, Matrix Analysis, Cambridge University Press, Cambridge, UK, 1985.

[2] C. Altafini, "Stability analysis of diagonally equipotent matrices," Automatica, vol. 49, no. 9, pp. 2780-2785, 2013.

[3] H. Tin-Zhu, "Stability criteria for matrices," Automatica, vol. 34, no. 5, pp. 637-639, 1998.

[4] L. Gao, D. Wang, and G. Wang, "Further results on exponential stability for impulsive switched nonlinear time-delay systems with delayed impulse effects," Applied Mathematics and Computation, vol. 268, pp. 186-200, 2015.

[5] L. Gao and D. Wang, "Input-to-state stability and integral inputto-state stability for impulsive switched systems with time-delay under asynchronous switching," Nonlinear Analysis: Hybrid Systems, vol. 20, pp. 55-71, 2016.

[6] L. Gao, D. Wang, and G. Zong, "Exponential stability for generalized stochastic impulsive functional differential equations with delayed impulses and Markovian switching," Nonlinear Analysis: Hybrid Systems, vol. 30, pp. 199-212, 2018.

[7] X. Wang, H. Chen, and Y. Wang, "Solution structures of tensor complementarity problem," Frontiers of Mathematics in China, vol. 13, no. 4, pp. 935-945, 2018.

[8] T. Dursun and S. Kirkland, "A sequence of upper bounds for the Perron root of a nonnegative matrix," Linear Algebra and its Applications, vol. 273, pp. 23-28, 1998.

[9] G.-X. Huang, F. Yin, and K. Guo, "The lower and upper bounds on Perron root of nonnegative irreducible matrices," Journal of Computational and Applied Mathematics, vol. 217, no. 1, pp. 259267, 2008.

[10] D. B. Szyld, "A sequence of lower bounds for the spectral radius of nonnegative matrices," Linear Algebra and its Applications, vol. 174, pp. 239-242, 1992.

[11] G. Wang, G. Zhou, and L. Caccetta, "Z-eigenvalue inclusion theorems for tensors," Discrete and Continuous Dynamical Systems - Series B, vol. 22, no. 1, pp. 187-198, 2017.

[12] W. E. Arnoldi, "The principle of minimized iteration in the solution of the matrix eigenvalue problem," Quarterly of Applied Mathematics, vol. 9, pp. 17-29, 1951.

[13] Z.-x. Jia and L. Elsner, "Improving eigenvectors in Arnoldi's method," Journal of Computational Mathematics, vol. 18, no. 3, pp. 265-276, 2000.

[14] W. Bunse, "A class of diagonal transformation methods for the computation of the spectral radius of a nonnegative irreducible matrix," SIAM Journal on Numerical Analysis, vol. 18, no. 4, pp. 693-704, 1981.

[15] F. Duan and K. Zhang, "An algorithm of diagonal transformation for Perron root of nonnegative irreducible matrices," Applied Mathematics and Computation, vol. 175, no. 1, pp. 762772, 2006.

[16] Y. Wang, L. Caccetta, and G. Zhou, "Convergence analysis of a block improvement method for polynomial optimization over unit spheres," Numerical Linear Algebra with Applications, vol. 22, no. 6, pp. 1059-1076, 2015.

[17] C. Wen and T.-Z. Huang, "A modified algorithm for the Perron root of a nonnegative matrix," Applied Mathematics and Computation, vol. 217, no. 9, pp. 4453-4458, 2011.

[18] H. Chen and Y. Wang, "A family of higher-order convergent iterative methods for computing the Moore-Penrose inverse," Applied Mathematics and Computation, vol. 218, no. 8, pp. 40124016, 2011.

[19] L. Collatz, "Einschliessungssatz fur die charakteristischen Zahlen von Matrizen," Mathematische Zeitschrift, vol. 48, pp. 221-226, 1942.

[20] Y. Wang, K. Zhang, and H. Sun, "Criteria for strong H-tensors," Frontiers of Mathematics in China, vol. 11, no. 3, pp. 577-592, 2016.

[21] Y. Wang and G. Wang, "Two S-type Z-eigenvalue inclusion sets for tensors," Journal of Inequalities and Applications, Paper No. 152, 12 pages, 2017.

[22] R. J. Wood and M. J. O’Neill, “An always convergent method for finding the spectral radius of an irreducible non-negative matrix," The ANZIAM Journal. The Australian \& New Zealand Industrial and Applied Mathematics Journal, vol. 45, pp. 474485, 2007.

[23] R. J. Wood and M. J. O'Neill, "Finding the spectral radius of a large sparse non-negative matrix," The ANZIAM Journal. The Australian \& New Zealand Industrial and Applied Mathematics Journal, vol. 48, pp. 330-345, 2007. 
[24] K. Zhang and Y. Wang, "An H-tensor based iterative scheme for identifying the positive definiteness of multivariate homogeneous forms," Journal of Computational and Applied Mathematics, vol. 305, pp. 1-10, 2016. 


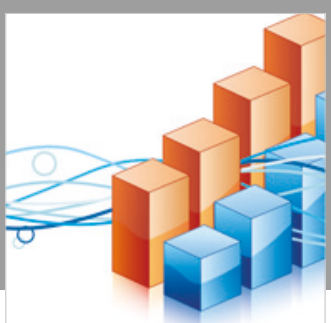

Advances in

Operations Research

\section{-n-m}
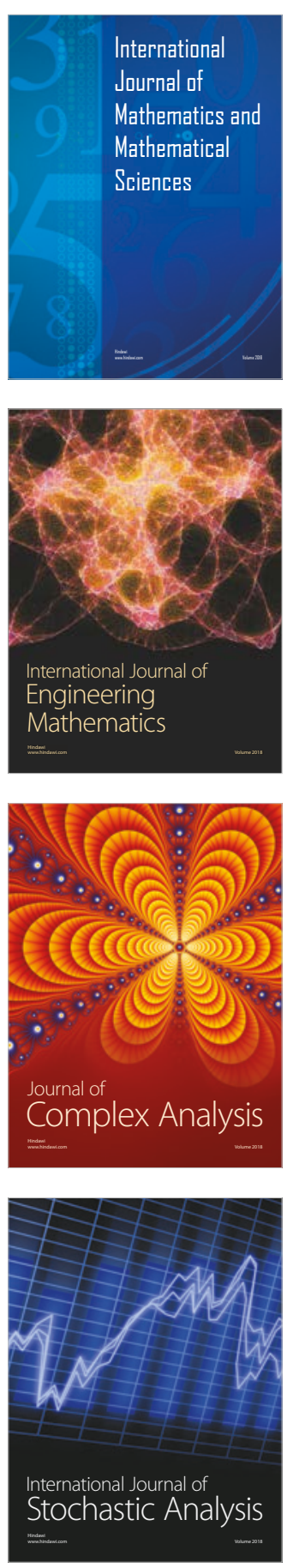
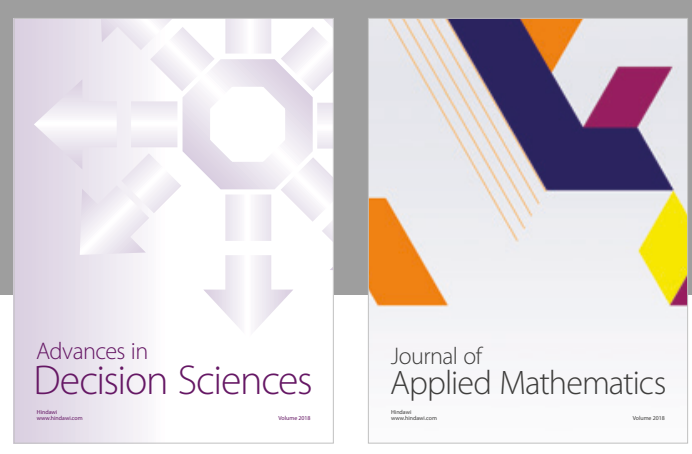

Journal of

Applied Mathematics
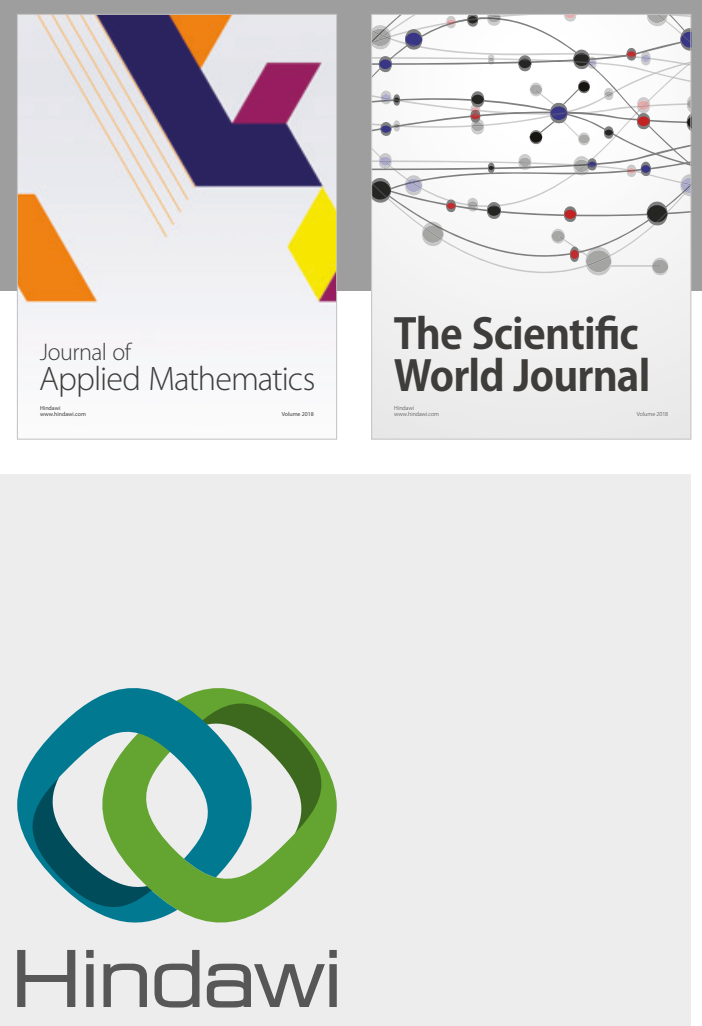

Submit your manuscripts at

www.hindawi.com

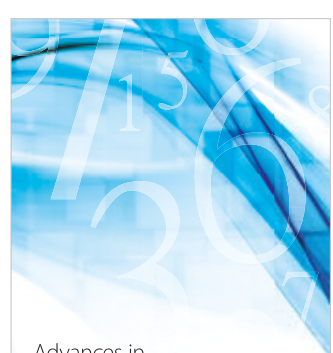

Advances in
Numerical Analysis
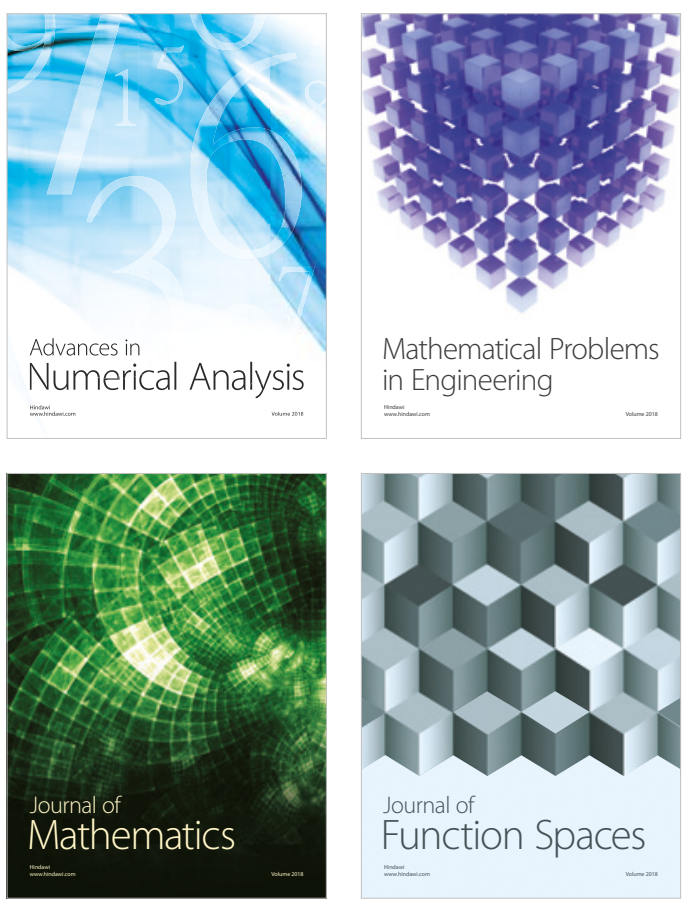

Mathematical Problems in Engineering

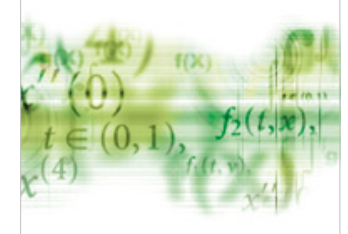

International Journal of

Differential Equations

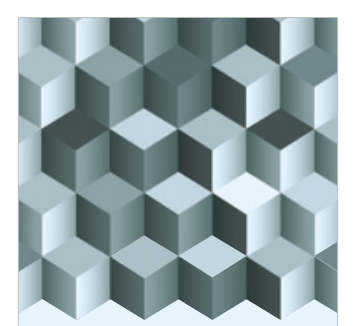

Journal of

Function Spaces

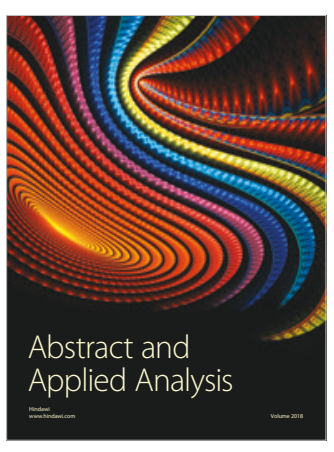

The Scientific

World Journal



Journal of

Probability and Statistics
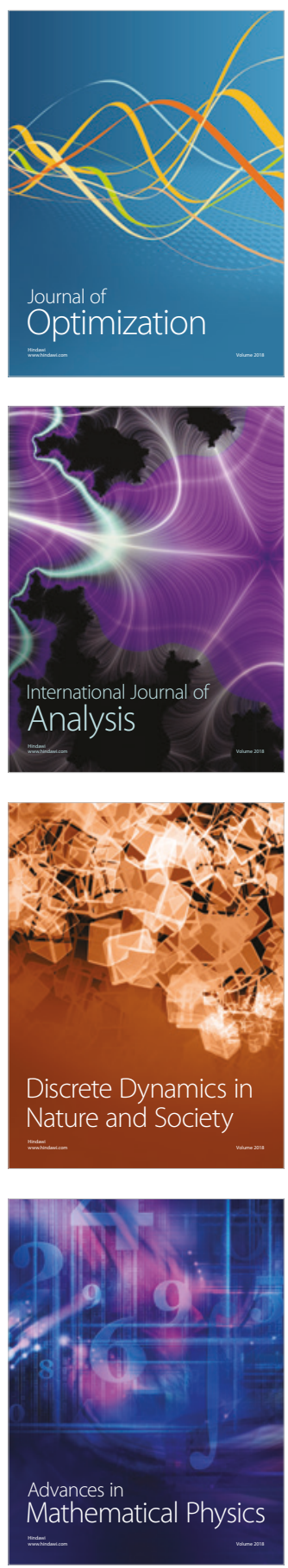